\title{
Mapping Collaboration in Open Source Geospatial Ecosystem
}

\section{Jianhua Shao}

Horizon Doctorial Training Centre University of Nottingham

\section{Suchith Anand}

Centre for Geospatial Science

University of Nottingham

\section{Mike J. Jackson}

Centre for Geospatial Science

University of Nottingham

\section{George Kuk}

Nottingham University Business School University of Nottingham

\section{Jeremy G. Morley}

Centre for Geospatial Science

University of Nottingham

\section{Tyler Mitchell}

Open Source Geospatial Foundation

Address for correspondence: Jianhua Shao, Horizon Doctoral Training Centre, School of Computer Science, Jubilee Campus, University of Nottingham, Nottingham, NG8 1BB, UK, Email: psxjs4@ nottingham.ac.uk 


\section{Abstract:}

Over the last decade, there has been a tremendous growth and exploitation of open source geospatial software and technologies. A combination of factors is driving this momentum including the contributions made by hundreds of OSGeo developers and the leading role played by the Open Source Geospatial Foundation (OSGeo), aiming primarily to support and promote the collaborative development of open source geospatial technologies and data. This paper seeks to map out the social history of collaborative activities within the OSGeo ecosystem. We used the archival logs of developers' contributions, specifically looking for boundary spanning activities where contributions crossed multiple projects. The analysis and visualization of these activities allow us to have a better understanding of the role of boundary spanning in the resourcing of each project, the incubation mechanism advocated by OSGeo, and the significance of the social interrelatedness among projects. The data consisted of the subversion (SVN) commit history made by individual developers in the programming code repository. We applied several network analytical and visualization techniques to explore the data. Our findings indicate that more than one in seven developers spanned multiple projects which potentially had the effects of shaping the projects' directions, and increased knowledge flow and innovation. Also the OSGeo's incubation mechanism provided an important encouragement for boundary spanning and increased knowledge sharing. By studying the social history of contributions, further tools can be developed in future to assist tracking of the social history, and make developers mindful of the significance of the interdependence among projects and hence continuously contribute to the healthiness of the OSGeo ecosystem.

Keywords: Open Source, OSGeo, Social Network, Collaboration, Ecosystem 


\section{Introduction:}

Open source development attracts a great number of participants from various backgrounds. Participants perform different roles to push forward the development cycles including code developers, software testers, policy makers, and normal users (Mockus et al. 2000). They contribute to and influence development within open source communities, and themselves benefit by being involved in this ecosystem (Lerner and Tirole 2002). Open source geospatial communities are a large group showing rapid growth in the last decade (Steiniger and Bocher 2009). A more recent development among some communities is to have a formalised organisation to help assist projects developing under its umbrella. In the geospatial open source domain, this organisation is the Open Source Geospatial Foundation (OSGeo).

$\mathrm{OSGeo}^{1}$ is a non-profit, non-governmental organization whose mission is to support and promote the collaborative development of open geospatial technologies and data (Mitchell 2010). The foundation was formed in February 2006 to provide financial, organizational, and legal support to the broader free and open source geospatial community. It also serves as an independent legal entity to which community members can contribute code, funding and other resources, secure in the knowledge that their contributions will be maintained for public benefit. OSGeo provides a common forum and shared infrastructure for improvising cross-project collaboration.

OSGeo provides an umbrella organisation with multiple sub-projects applying to join (Mitchell 2010). It is different from other open source communities in terms of the governance structure and the use of incubation as a decision mechanism. The

1 http://www.osgeo.org/ 
decisions to sanction new projects are largely subject to an incubation mechanism, where OSGeo incubators have the responsibility of preparing and recommending newly joined projects to the OSGeo Board of Directors (Christl 2010). The incubators essentially review applications and appoint mentors for incubating projects. Yet there is no mandate that incubated projects have to work collaboratively with other existing OSGeo projects as each project can develop separately and in some instances, compete with others for developers. Although anecdotal evidence exists suggesting that interaction among projects is common, and that innovation and knowledge can be transferred and shared across OSGeo projects, it is not clear whether the interactions are largely restricted to the technical dependency among projects, for example, similar projects using the same library of codes. We know less about the social aspects of OSGeo ecosystem relative to the technical interrelatedness of projects (Sanz Salinas 2009).

It is vital to understand that the collaboration among projects can positively affect the healthiness of the ecosystem. Independent development can only deter wider adoption by the industry, as it can have deleterious effects. For example, in assessing the impact of developer activities on the known security vulnerabilities in the Red Hat Enterprise Linux $4 \mathrm{kernel}$, Meneely and Williams (2009) found that too many independent contributors can dilute the focus and lead to submission of vulnerable software patches. It is found that open source projects that are developed in tandem with each other are more likely to be more innovative and attractive to developers (Kuk, 2010). This paper seeks to examine the interrelatedness among projects based on the ways developers contribute to projects. Our premise is that having developers 
that contribute to and crisscross multiple projects can increase the liquidity of knowledge flow and sharing, and collectively move innovation forward.

The remainder of the paper is set out as follows. Section 2 outlines the data collection processes giving details about the database structure and a summary of the SVN data analysed. Data analysis is described in section 3, with results from social network analysis, development effort overlap, boundary spanning and the impact of OSGeo incubation. Limitations and future work are presented in section 4. The paper's conclusions are presented in section 5 . 


\section{Data collection}

Due to the openness, open source participants leave rich digital footprints online, especially for those people who directly contribute to the source code of a project. For example, they make comments to reflect the change of code; they also identify the places that code could be improved; they can even discuss how the code may be used in practice (Kogut and Metiu 2001). This phenomenon also occurs in the OSGeo community. Our aim has been to collect data that reflects user activities when contributing to the development of software within OSGeo. Three publicly available data sources were identified for deriving contribution metrics: Subversion (SVN) code repository commits; mailing lists; and issue trackers (Gutwin, Penner et al. 2004). SVN commits are used to track individual and aggregate contributions of source code and are taken directly from a project's source code repository. Most projects in OSGeo use SVN to track and manage source code development, and it makes it easy to publicly fetch for analysis (see Table 1). The open source community also uses mailing lists to communicate with each other for a broad range of purposes. Each project has its own set of mailing lists and the whole OSGeo community has a general set of mailing lists for cross-project or organisational discussion. Issue trackers are another data source available to study the interaction between community members. People report, create and assign bugs, tasks and feature requests within communities, at various scales, from branches to project to community. Here we focus on SVN commits as our data source, as they are easiest to access and understand. Further research will consider the other two types of data sources.

SVN commits reflect the development history of an individual project. They also reflect development interaction between users from different projects (Perera et al. ; 
Sowe et al. 2008). SVN is a software versioning and a revision control system (Collins-Sussman et al. 2004). Developers use SVN to maintain current and historical versions of files in projects, such as source code and documentation. SVN maintains versioning for file directories, source files, and file metadata. There are many other versioning control tools, like CVS (Cederqvist and Pesch 1993) and Git (Torvalds and Hamano 2010), but SVN is, historically, the most popular (Hammond et al. 2011). The open source community has used SVN widely; nearly all OSGeo projects use SVN or provide an SVN mirror. A commit, in the context of SVN, refers to submitting the latest changes of the source code to the repository, making these changes part of the head version of the repository and then allowing them to be synchronised with other users. Therefore, people make commits when they make any change to the source code, and the changing records would reflect how that project's source code has developed. Thus it is a great source to analyse the process of collaborative coding in OSGeo.

There are three main types of SVN commits which are, essentially, three separate folders in each project: trunk, tags and branches (Collins-Sussman, Fitzpatrick et al. 2004). The trunk folder holds the main body of development, originating from the start of the project until the present. It generally has all the newest features. The tags folder is a point in time on the trunk or a branch that individual developers wish to preserve. The two main reasons for preservation would be that either this is a major release of the software, or this is the main stable point of the software before major revisions on the trunk were applied. The branches folder holds a copy of code derived from a certain point in the trunk that is used for applying major changes to the code while preserving the integrity of the code in the trunk. It always represents a smaller 
release and new features. SVN uses a standard method for locating those three sources. It all starts with a root repository URL, and then follows with the resource name. Therefore, once we know one resource address, it is easy to figure out the other resource addresses. For example, the commit path for the PostGIS project's trunk is http://svn.osgeo.org/postgis/trunk

Each SVN commit message is well structured and we can retrieve it as a consistent rich data source. SVN Commits are stored internally within the repository but can be output in XML format using a command similar to the following:

$$
\text { svn log svn_path }-v-x m l>\text { output_file_name }
$$

Each commit includes data such as: revision, author, date, path and message. Table 3 shows an example from the deegree project. Each commit is a logentry message. Each logentry will show the unique revision ID within the project, it will also record who made the change to the code and what time it happened. SVN commits also record what files have been changed and the actions, such as adding, deleting or modifying a file. Developers usually leave a meaningful, human-readable message for each change as well, which provides the opportunity to do further text mining in future research. All SVN commits data sources use this same format to host the message, regardless of whether it is trunk, tags or branches. The only differences are where those commits come from within the resource URL, and the $m s g$ tag which will have a different message. As with the message demonstrated below, we can easily understand that it is a commit from a tag by looking at the msg element.

We designed and implemented a data collection server to aggregate statistics on the public development activities within the OSGeo community. Based on the SVN 
commits standard, we designed and implemented a database as shown in Figure 1. It was designed to be easy to add new commit logs and provide a quick query searching. This database is also easy to adapt when we study new data sources. The design is implemented using SQLite $3^{2}$ for its flexibility. The data collection server was written in the Python ${ }^{3}$ language and has been running and stable for more than three months from February 2011. At this stage of the project we only studied the mature projects that had already graduated from OSGeo Incubation. At the time of writing this paper, the database file had passed $300 \mathrm{MB}$ in size with more than $300,000 \mathrm{SVN}$ commits records. These records were historic information from the SVN repositories of each OSGeo projects.

\footnotetext{
${ }^{2}$ http://www.sqlite.org/
}

${ }^{3}$ http://www.python.org/ 


\section{Data analysis}

Through some basic analysis of the data, we found there are about 700 developers who have been actively contributing to the source code development and left their name on it. It is reasonable to expect that there are a much larger number of people who use the software but who do not contribute to the source code. More importantly, we find about 1 in 7 of the active developers contribute to 2 or more projects (see Figure 2). About $80 \%$ of the developers working on multiple projects only work on 2 projects. A small number of developers work on more than 5 projects. We even find one developer working for 7 different projects and who is also active in OSGeo community (see Figure 2). To study the social collaboration, we are interested in analysing those people who work on multiple projects. We describe the analysis process and results below from four successive angles: social network analysis; development effort overlap; boundary spanning; and the impact of OSGeo incubation.

\subsection{Social Network Analysis}

We have studied the OSGeo community by first adapting a social network analysis method (Scott 2000; Lopez-Fernandez et al. 2004). Social network analysis applies network theory to social relationships. The social network is made up of individuals or organisations as nodes, which are tied or connected by one or more specific types of interdependency, such as common interest, knowledge sharing. The ties are the network edges. Nodes are the individual actors within the networks, and ties are the relationship between the actors. The resulting graph-based structure is often very complex and there can be many kinds of ties between the nodes. Research in a number of academic fields has shown that social networks operate on many levels (Barnes 1954; Barthes and Duisit 1975), from families up to the level of nations, and 
play a critical role in determining the way problems are solved, organisations are run, and the degree to which individuals succeed in achieving their goals. The small world phenomenon (Watts and Strogatz 1998) is the hypothesis we are interested in for this research, saying that the chain of social acquaintances required to connect one arbitrary person to another arbitrary person anywhere in the world is generally short. In this theory, the network comprises a number of cohesive groups or connected components. They are connected to each other via bridges or "short-cuts".

Social networks may reflect on knowledge innovation and transformation (MüllerProthmann 2006). The shape of a social network helps determine a network's usefulness to its individuals. Smaller, tighter networks can be less useful to their members than networks with lots of loose connections (weak ties) to individuals outside the main network (Granovetter 1973). More open networks, with many weak ties and social connections, are more likely to introduce new ideas and opportunities to their members than closed networks with many redundant ties. In other words, a group of friends who do things with each other already only share the same knowledge and opportunities. A group of individuals with connections to other social groups is likely to have access to a wider range of information. It is better for individual success to have connections to a variety of networks rather than many connections within a single network. Similarly, individuals can exercise influence, or act as brokers, within their social networks by bridging two networks that are not directly linked. The OSGeo community has a social network that is interesting to study. It is, firstly, working as a substantial foundation with many sub-projects, and then each project attracts many people who contribute. Meanwhile, many people are communicating across different projects, even outside of the OSGeo community. The 
social network relationship in OSGeo should naturally show diversity, but they all aim for the same target: to encourage the use and development of OSGeo software. These reasons have attracted us to study the social collaboration within OSGeo.

\subsection{Development effort overlap}

We can view the connection between projects by looking at the relationship of people to projects. People are definitely connecting to a project if they contributed to project source code development - they have to understand existing code before committing code. Therefore, individual users may also establish connections with each other if they contribute to the same part of the source code with operations like create, copy, delete, rewrite and modify. Based on this assumption, we created Figure 3 which is a diagram based on historic SVN commit data that represents the relationship of people to projects. Each green dot represents an individual developer and each blue dot means one individual project. The edge, or tie, bridging a green dot and a blue dot means the person has contributed to the project. This figure does not include any edge weights, but this is analysed in section 3.3 with weights calculated from how much contribution a person has made to a project.

By looking at the diagram (Figure 3), we can see that most developers concentrate on one project, but some of them spread their efforts across several projects. Unlike other kinds of contribution, code contribution requires professional coding skills, good understanding of existing source code, and much time invested in the application area. Therefore, concentration on one project makes development work much easier, and this is common in traditional open source development. However, it is slightly different in OSGeo, as nearly all OSGeo projects have multiple developers working 
on other projects and those developers connect different projects together. Those developers distribute their development time into different projects, and more importantly we find that they transfer knowledge and innovation between projects. For example, the GeoServer project is partly built based on the GeoTools project. There are a number of developers working on both projects. They would import libraries from GeoTools project into GeoServer projects, like data access, rendering and referencing. Meanwhile, those developers would also transform functional demands from GeoServer project into GeoTools project, like efficiency of data rendering, supporting for new data format. Because OSGeo is a big umbrella on top of a number of open source projects, it provides an opportunity for people to communicate and share new ideas and information, so it makes this kind of overlapping development effort more common compared to other open source communities, like the Linux Foundation ${ }^{4}$ and the Apache Foundation ${ }^{5}$. The Linux Foundation is a single-source open source community where most development efforts are around the Linux operating system. Even though there are several projects on going, it is still considered as a single project because those projects integrate with each other so much (Welsh 1994). Apache Foundation is the opposite, it also holds many projects, but those projects are loosely connected (Roberts et al. 2006). OSGeo's operation lies in the middle. It has many projects, but those projects are not considered as components of a giant system, they are all excellent in their own application areas. Those projects are also not loosely connected, as they all address geospatial subjects. This unique phenomenon significantly encourages the communication between projects in the OSGeo community.

4http://www.linuxfoundation.org/

5 http://www.apache.org/ 
In addition, this network diagram (Figure 3) also illustrates a cohesion distance between the projects (represented as blue dots in the diagram). The cohesion distance is represented by the distance between the pairs of project dots. This distance is calculated based on Harel-Koren Fast Multiscale algorithm (Harel and Koren 2001) to present the interaction of development between projects. Projects in the middle of the diagram, which have more open connections to other projects, have a much shorter distance than individual, closed projects away from the middle of diagram. Openness here means how much connection it has to others, which could be project software dependencies and also participants' involvement. The open projects normally also have more participants involved in development. OSGeo projects have dependencies between projects, for example the relationship between the GeoServer and GeoTools projects, and so we see much interaction between clustered projects. Yellow and purple dots in the diagram represent GeoTools and GeoServer project. The GeoServer project is heavily dependent on data access, rendering and referencing system libraries from GeoTools. GeoServer developers have a policy to contribute back to GeoTools whatever code has the potential to be shared with other projects. We can also view this relationship from the diagram. Their distance is very short and many developers connect both projects. This contributes greatly to boundary spanning which will be discussed more in sections 3.3.

Because of the project dependencies and similarity in objectives between projects, participants have more opportunities to contribute within OSGeo, as they can more easily become involved in different projects. In the middle of the diagram, some developers (represented by green dots) contribute to several projects, acting as a hubs 
for project-to-project connectivity. Those projects are closer to each other and they normally have more contributors. In turn, such short project distances would also increase the possibility of people becoming involved in different projects. Because those projects share similarities in development the developers should find it easier to apply their knowledge in a different project. The diagram also shows that people working on different projects are more commonly clustered in the middle of diagram, close to the projects with more open connectivity. The red arrows are an example to demonstrate that one person is committing to seven projects and is near to this central cluster. It is interesting to see in section 3.3 what value those people make by connecting different projects.

\subsection{Boundary spanning}

Boundary spanning describes the phenomena where people divide their time between different projects, linking the development between different projects across the "boundaries" of projects (Daft 2009). The linking could be information exchange, relationship building, and using "boundary objects" as a way to create shared meaning and trust across the boundary (Williams 2002). It has been studied several times as a topic in leadership (Yip et al. 2009). Here we have applied this for the first time to an open source community. From the literature, boundary spanning is concerned with the detection of information. It has two primary roles. One is to detect information and bring it into the organisation. The second is to send information into the environment, presenting the organisation in a favourable light.

From the analysis in the last section, we find that boundary spanning is common in the OSGeo community. Around 1 in 7 OSGeo developers have experienced, or are 
experiencing, boundary spanning by contributing to multiple projects. Most of them have a primary project to work on, meaning that they will contribute most of their time to a single primary project. However, for many reasons, they spend part of their time on several other projects. Boundary spanners serve strategic roles in OSGeo projects by gathering critical information, obtaining feedback and perception from the external environment through their networks and then interpreting and translating that information back into their primary projects. For example, some boundary spanners in GeoServer projects would bring feedback and expectation back to their primary project, GeoTools, to support the development of the additional library functions necessary for GeoServer. Ultimately, if the boundary spanner is effective, the process can lead to innovation in strategy, processes or products (Ansett 2005). We summarise the key activities of the boundary spanner in OSGeo as:

1. Creating internal and external networks for open source projects,

2. Acting as the identifier for solution development,

3. Translating the knowledge back into the project culture,

4. Influencing and educating internal and external users of projects.

Even though boundary spanning brings many benefits, boundary spanning still has trade-offs, especially in OSGeo code contribution. Because code contribution takes professional skills and project familiarity, it is a time-consuming activity. If people work on too many projects, they may not be able to contribute as significantly on a single project or even all projects at an average level. However, when people concentrate on single projects, they more easily sharpen their skills within the project. Open source collaboration is different to other commercial organisation; developers may be more readily motivated by reputation. Boundary spanning connects to such a 
motivation, helping to enumerate a developer's contribution to innovation and knowledge transformation across projects. In practice, boundary spanning would be evaluated indirectly over time. Studying OSGeo's boundary spanning can help us understand the open source development organisation and stimulation. Table 3 and Figure 4 demonstrate the metrics for one boundary spanning developer who has been working on 7 projects. From the statistics, we can see his primary project is GDAL, but he also spends significant effort on MapServer and other projects. GDAL (Geospatial Data Abstraction Library) is an access and translation library for geospatial data formats. MapServer supports rendering maps from various data sources, using, in particular, the GDAL library. Similar to other projects, this person boundary spans different projects to transform the knowledge and innovation among them, thereby reducing development costs and increasing the development speed and code reuse across projects.

\subsection{Incubation}

The incubation process also distinguishes the OSGeo community from other open source communities. The purpose of the OSGeo incubation process is to ensure projects follow the basic official OSGeo guidelines on Open Source software development (Christi 2010). It means all OSGeo projects have a successfully operating, open and collaborative development community to mentor each project through the incubation process. Open Source software projects originate from very different backgrounds and cover all types of programming environments. Some OSGeo projects have been Open Source right from the start and evolved over many years like the GRASS project that started more than 25 years ago. Other projects now published under an Open Source license were initially managed by a single 
commercial entity, for example MapGuide Open Source where Autodesk changed the development and governance structures so the code was opened to the broader community. To become a successfully incubated project, the project team should manage themselves, striving for consensus and encouraging participation from all contributors, from beginning users to advanced developers. Contributors are the scarce resource. OSGeo counts successful incubated projects and encourage those projects to share their contributors Projects are also encouraged to adopt open standards and collaborate with other OSGeo projects; they are responsible for reviewing and controlling their code bases to ensure the integrity of the open source baselines.

By measuring the SVN commits of all OSGeo projects, based on timeline, we can prove that most OSGeo projects enjoy the benefits from OSGeo incubation with resource sharing. An individual project manages its development roadmap, but also shares the communication channel with other projects within the OSGeo ecosystem. By looking at Figure 6, we can see that most OSGeo projects have more active participants during the incubation. It leads to a significant increase of SVN commits which means direct contributions to coding. Meanwhile, by looking at the release of a product, we can also see a larger increase in output appearing during the period right before incubation. When OSGeo projects graduate from incubation there are two patterns for continued project development. One pattern is to continue with high, or even higher, code development outputs through the development of further functionality. The other pattern is of decreasing output as most bugs and functions have been developed before graduation from incubation. Some projects started a long time before incubation through OSGeo was available. With a successful set of 
projects which had graduated from incubation, OSGeo attracted many other projects that joined in incubation, which contributes to the continued growth of the resources available through a form of snowballing effect. We also measured the ratio of coding contribution and project output, especially for projects that have dependencies with each others, like GeoServer and GeoTools. We find those projects have similar development output rates. This makes us confident to believe that innovation, knowledge interaction and resource sharing is common in OSGeo incubation, and any boundary spanner would perform important bridges for this targeting (see Figures 5 \& $6)$. 


\section{Limitations \& Future work}

The aim of this project is to study the social collaboration of the OSGeo community. Even though this paper only introduces the project and the initial work being carried out, further research activities have been planned and more activities will be introduced as data collection and analysis improve. At present, we only collect SVN commits as a starting point to study the social collaboration in development. SVN commits records the history of project code evaluation. Yet SVN commits cannot help us measure the project delivery in a more precise way. There are three reasons for this.

1. Because, in practice, the SVN commits do not necessarily identify real people. We can only find the SVN commits' user names but this is not a match to a real name and we cannot guarantee that multiple users don't share the same account. SVN commit accounts are not open to all people who want to contribute to the codes. Therefore, we are missing some people who may want to contribute but are not recorded in the central repository.

2. Due to the different understanding of output standards. Small releases are treated as important outputs in some projects. Because a version number is controlled by a project's administer, there is a wide variation between projects in the understanding of version division. We believe future study is needed to explore this further. One uncompleted analysis to be reported in a further paper is temporal emergence. This is to study the patterns in how people spend time on open source development. It helps to understand the development trajectory of open source. 
3. Due to limitation in the scope of the data. SVN commits only records people who directly contribute to coding, therefore we can only track the coders in OSGeo. However, there are many people who would contribute to the development of source code indirectly, like source code testers and product users, who help to identify the place where source code should be improved. OSGeo is not only code developer driven, but also influenced by other contributors. Therefore, SVN commits is only a starting point as a data source. We plan to collect mailing list and issue tracker data to enrich our data source in the next step. The study model introduced in this paper can also be applied for studies with those two sources. The particular value of this further research is to build a model to visualize the evolution of open source communities like OSGeo. 


\section{Conclusions}

This paper introduced a multidisciplinary research project at the University of Nottingham to study social collaboration networks in Open Source Geospatial (OSGeo) communities. It started by introducing the OSGeo community which is an international foundation with more than 20 collaborative open source projects. We then described the data collection on SVN commits from each mutual project to reflect user activities in OSGeo. We have built a server written in Python to capture a synchronous picture of the development evolution of the whole OSGeo community. Based on existing data, we adopt social network analysis methods to explore the data. We find that around 1 in 7 of developers is boundary spanning their development effort into multiple projects. Most of those who boundary span work on two projects, but we find some boundary spanners are able to manage contributions to a larger numbers of projects. Core developers could sharpen their professional contribution within projects, but we find boundary spanners can contribute innovation transformation and knowledge sharing among OSGeo community. OSGeo incubation provides a central resource-sharing place that increases the possibility of boundary spanning. This study helps us understand the social collaboration of development activity evolution. The model introduced in this paper could be applied to other public data sources, such as mailing lists and issue trackers. This project plans to continue to build the index model to reflect the social evolution within open source geospatial software ecosystem. 


\section{Acknowledgements}

The project is supported by the Horizon Doctoral Training Centre at the University of Nottingham (RCUK Grant No. EP/G037574/1), and was carried out through internship funding through the Open Source Geospatial Lab at the Centre for Geospatial Science. We also thank the Ordnance Survey for funding for Suchith Anand's research through the Future Data project. 


\section{References}

Ansett, S. 2005. Boundary spanner: The gatekeeper of innovation in partnerships.

Barnes, J. A. 1954. "Class and committees in a Norwegian island parish." Human relations 7(1): 39 .

Barthes, R. and L. Duisit 1975. "An introduction to the structural analysis of narrative." New Literary History 6(2): 237-272.

Cederqvist, P. and R. Pesch 1993. "Version management with CVS." Signum Support AB.

Christi, A. 2010. "The State of OSGeo and the global SDI."

Collins-Sussman, B., B. W. Fitzpatrick, et al. 2004. Version control with subversion, O'Reilly Media, Inc.

Daft, R. L. 2009. Organization theory and design, South-Western Pub.

Granovetter, M. S. 1973. The strength of weak ties. The American journal of sociology 78(6): 1360-1380.

Gutwin, C., R. Penner, et al. 2004. Group awareness in distributed software development, ACM.

Hammond, J. S., M. Gilpin, et al. 2011. "The State Of Application Development In Enterprises And SMBs."

Harel, D. and Y. Koren 2001. A Fast Multi-scale Method for Drawing Large Graphs. Proceedings of the 8th International Symposium on Graph Drawing, Springer-Verlag: 183-196.

Kogut, B. and A. Metiu 2001. Open Source Software Development and Distributed Innovation. Oxford Review of Economic Policy 17(2): 248.

Kuk, G., 2010, Eyeballs, bugs, and releases in open source software, at European Conference on Information Systems, South Africa, June.

Lerner, J. and J. Tirole 2002. Some simple economics of open source. The journal of industrial economics 50(2): 197-234.

Lopez-Fernandez, L., G. Robles, et al. 2004. Applying social network analysis to the information in cvs repositories, Citeseer.

Mitchell, T. 2010. 2009 Annual Report-All Reports. OSGeo Journal 7(1): 28.

Meneely, A. and Williams, L. 2009. Secure open source collaboration: An empirical study of Linus' law. Paper accepted at the 16th ACM Conference on Computer and Communications Security, Chicago, November.

http://www4.ncsu.edu/ apmeneel/ccs221-meneely.pdf

Mockus, A., R. T. Fielding, et al. (2000). A case study of open source software development: the Apache server, Acm. 
Müller-Prothmann, T. (2006). "Leveraging knowledge communication for innovation." Framework, Methods and Applications of Social Network Analysis in Research and Development. Frankfurt a. M. et al.: Peter Lang.

Perera, D., J. Kay, et al. "Mining learners' traces from an online collaboration tool." Educational Data Mining: 60.

Roberts, J. A., I. Hann, et al. (2006). "Understanding the motivations, participation, and performance of open source software developers: A longitudinal study of the apache projects." Management Science 52(7): 984.

Sanz Salinas 2009 Panorama of the ecosystem of Open Source GIS. Available at https://confluence.prodevelop.es/display/pan/Panorama+del+ecosistema+de+Software +Libre+para+SIG+-+completo

Scott, J. (2000). "Social network analysis."

Sowe, S. K., I. Samoladas, et al. (2008). Are FLOSS developers committing to $\mathrm{CVS} / \mathrm{SVN}$ as much as they are talking in mailing lists? Challenges for Integrating data from Multiple Repositories.

Steiniger, S. and E. Bocher (2009). "An overview on current free and open source desktop GIS developments." International Journal of Geographical Information Science 23(10): 1345-1370.

Torvalds, L. and J. Hamano (2010). "GIT-fast version control system." URL http://git-scm. com.

Watts, D. and S. Strogatz (1998). "Small world." Nature 393: 440-442.

Welsh, M. (1994). "Cooking with Linux: Linux Leadership." Linux Journal 1994(2es): 13.

Williams, P. (2002). "The competent boundary spanner." Public Administration 80(1): 103-124.

Yip, J., C. Ernst, et al. (2009). "Boundary Spanning Leadership." 
List of Tables

\begin{tabular}{|c|c|c|c|c|c|}
\hline Project & SVN URL & Commits & Developers & $\begin{array}{l}\text { Developer } \\
\text { s on multi- } \\
\text { projects }\end{array}$ & $\begin{array}{l}\text { Boundary } \\
\text { spanning } \\
\text { rate \% }\end{array}$ \\
\hline deegree & $\begin{array}{l}\underline{\text { https://svn.wald.intevatio }} \\
\underline{\text { n.org/svn/deegree/ }}\end{array}$ & 9624 & 26 & 1 & 3 \\
\hline geomajas & $\begin{array}{l}\underline{\text { https://svn.geomajas.org/ }} \\
\underline{\text { majas/ }}\end{array}$ & 6915 & 16 & 3 & 18 \\
\hline GeoServer & $\begin{array}{l}\text { http://svn.codehaus.org/g } \\
\text { eoserver/ }\end{array}$ & 12103 & 58 & 29 & 50 \\
\hline MapBender & $\begin{array}{l}\text { https://svn.osgeo.org/ma } \\
\text { pbender/ }\end{array}$ & 7723 & 38 & 3 & 7 \\
\hline MapBuilder & $\begin{array}{l}\text { http://svn.codehaus.org/ } \\
\underline{\text { mapbuilder/ }}\end{array}$ & 3111 & 30 & 10 & 33 \\
\hline MapFish & $\begin{array}{l}\text { http://www.mapfish.org/s } \\
\text { vn/mapfish/ }\end{array}$ & 3758 & 31 & 14 & 45 \\
\hline MapGuide & $\begin{array}{l}\text { https://svn.osgeo.org/ma } \\
\text { pguide/ }\end{array}$ & 5072 & 59 & 18 & 30 \\
\hline MapServer & $\begin{array}{l}\underline{\text { http://svn.osgeo.org/map }} \\
\underline{\text { server/ }}\end{array}$ & 11089 & 53 & 18 & 33 \\
\hline OpenLayer & http://svn.openlayers.org/ & 4654 & 15 & 10 & 66 \\
\hline
\end{tabular}




\begin{tabular}{|c|c|c|c|c|c|}
\hline $\begin{array}{l}\text { GRASS } \\
\text { GIS }\end{array}$ & $\begin{array}{l}\text { https://svn.osgeo.org/gras } \\
\underline{\text { s/grass/ }}\end{array}$ & 39941 & 74 & 8 & 10 \\
\hline $\begin{array}{l}\text { Quantum } \\
\text { GIS }\end{array}$ & $\begin{array}{l}\text { https://svn.osgeo.org/qgis } \\
\underline{I}\end{array}$ & 14514 & 50 & 9 & 18 \\
\hline gvSIG & $\begin{array}{l}\text { http://subversion.gvsig.or } \\
\text { g/gvSIG/ }\end{array}$ & 31441 & 79 & 4 & 5 \\
\hline FDO & http://svn.osgeo.org/fdo/ & 5029 & 39 & 17 & 43 \\
\hline $\begin{array}{l}\text { GDAL/OG } \\
\mathrm{R}\end{array}$ & http://svn.osgeo.org/gdal/ & 21345 & 74 & 24 & 32 \\
\hline GEOS & $\begin{array}{l}\text { http://svn.osgeo.org/geos } \\
\underline{I}\end{array}$ & 3275 & 17 & 10 & 58 \\
\hline GeoTools & $\begin{array}{l}\text { http://svn.osgeo.org/geot } \\
\underline{\text { ools/ }}\end{array}$ & 24164 & 113 & 34 & 30 \\
\hline MetaCRS & $\begin{array}{l}\text { http://svn.osgeo.org/meta } \\
\underline{\mathrm{crs} /}\end{array}$ & 1991 & 30 & 21 & 70 \\
\hline OSSIM & $\begin{array}{l}\text { http://svn.osgeo.org/ossi } \\
\underline{\mathrm{m} /}\end{array}$ & 17600 & 27 & 5 & 18 \\
\hline PostGIS & $\begin{array}{l}\text { http://svn.osgeo.org/post } \\
\text { gis/ }\end{array}$ & 6324 & 23 & 9 & 39 \\
\hline $\begin{array}{l}\text { GeoNetwor } \\
\mathrm{k}\end{array}$ & $\begin{array}{l}\text { https://geonetwork.svn.so } \\
\underline{\text { urceforge.net/ }}\end{array}$ & 2831 & 17 & 1 & 5 \\
\hline
\end{tabular}

Table 1: The list of SVN Commits (October 1998 to April 2011). 


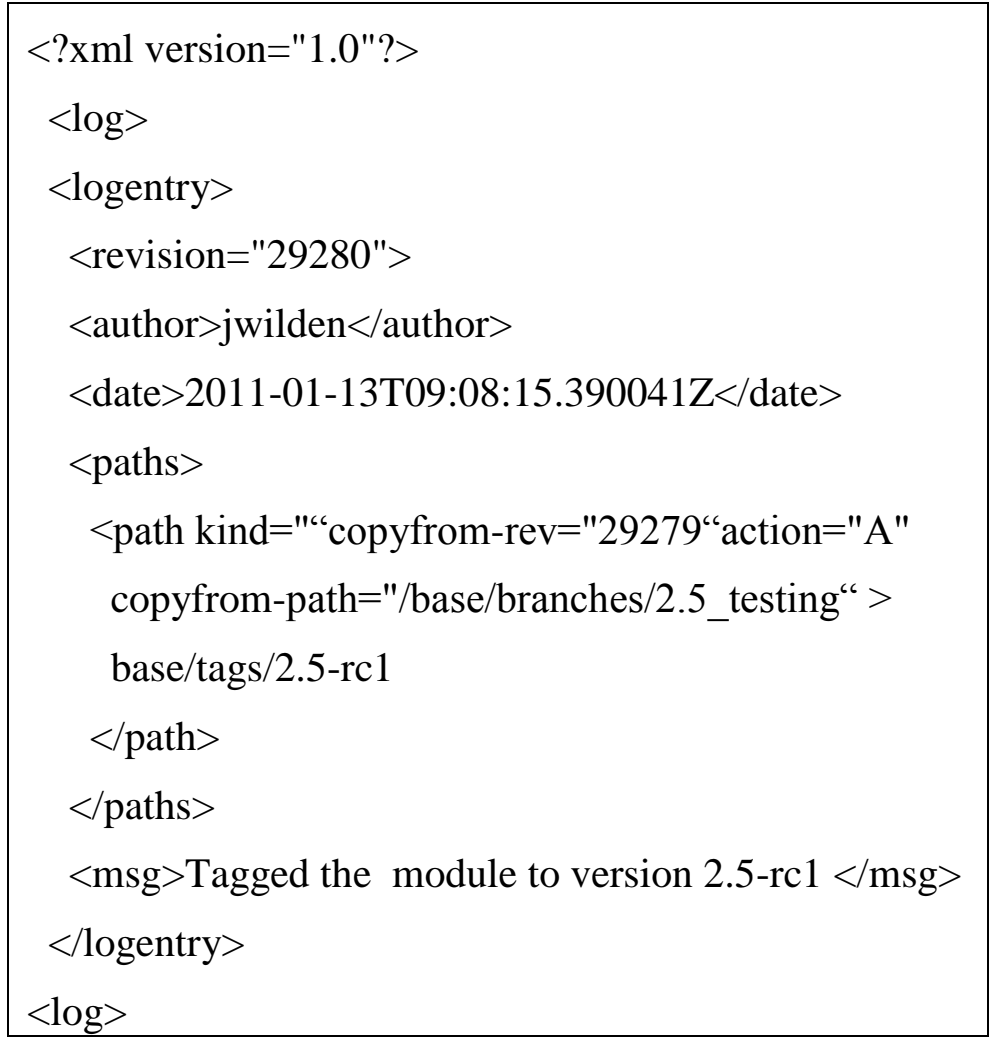

Table 2: an example to show how SVN Commits look 


\begin{tabular}{|l|l|}
\hline Project name & Revision count \\
\hline MapGuide & 1 \\
\hline MapServer & 398 \\
\hline Quantum GIS & 2 \\
\hline FDO & 69 \\
\hline GDAL/OGR & 2603 \\
\hline GEOS & 26 \\
\hline MetaCRS & 194 \\
\hline
\end{tabular}

Table 3: Table to show how a real developer distributes his development contribution into seven different projects. 


\section{List of figures}

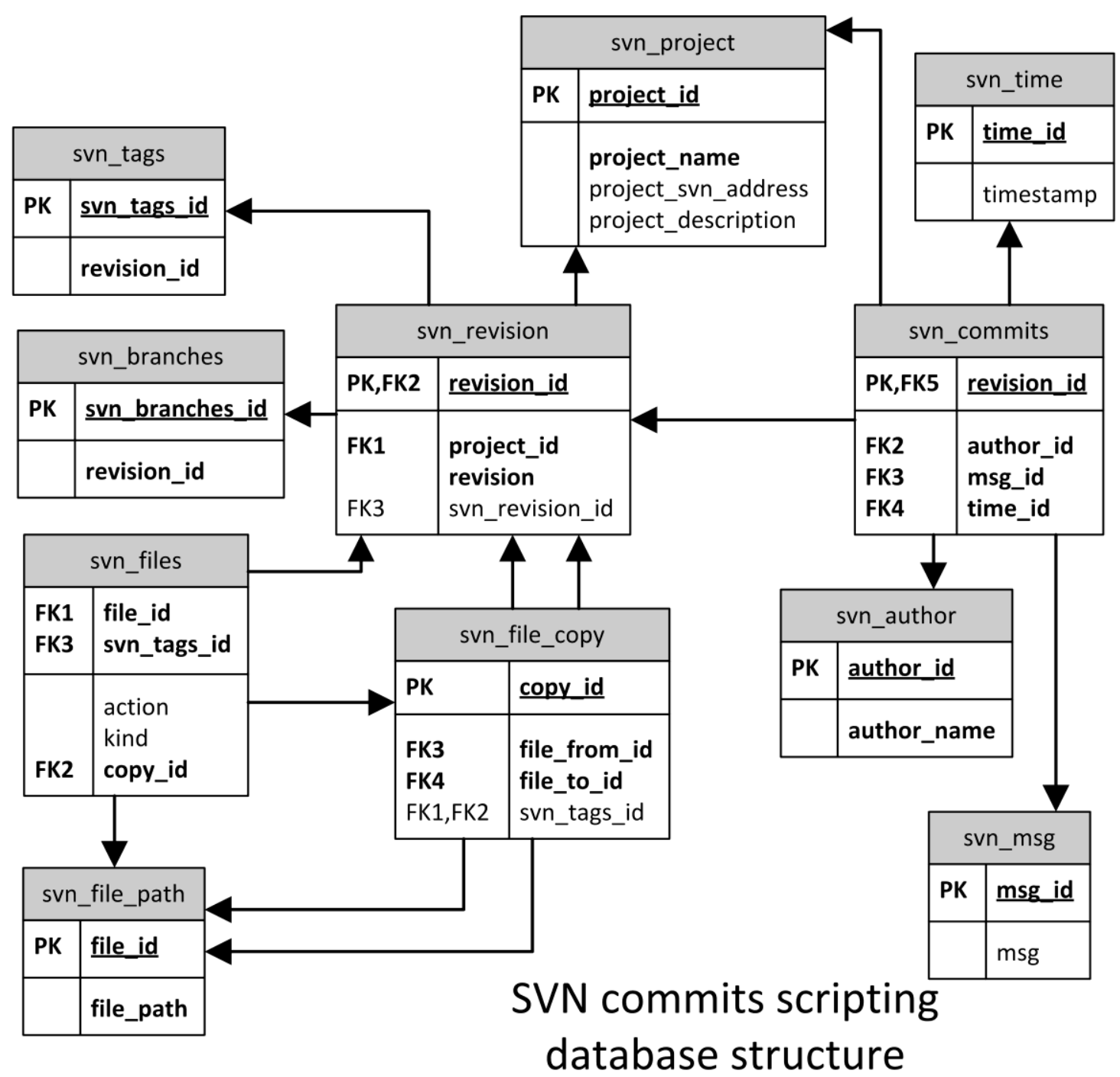

Figure 1: A schematic diagram of the SVN commits database 


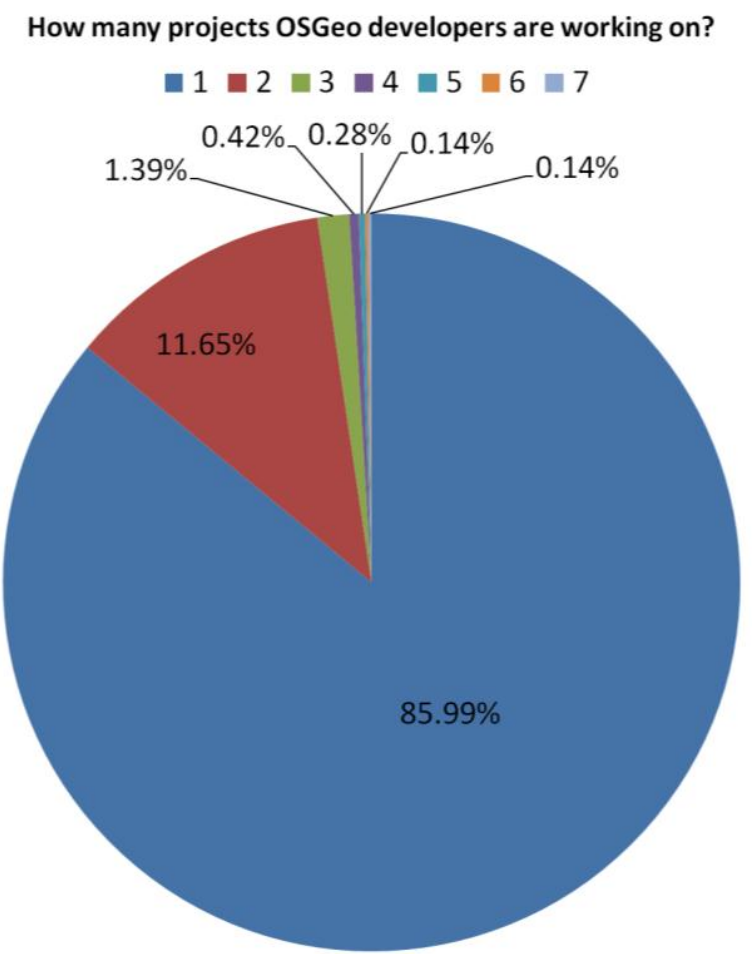

Figure 2: Distribution of developers by the number of OSGeo projects they contribute to. 


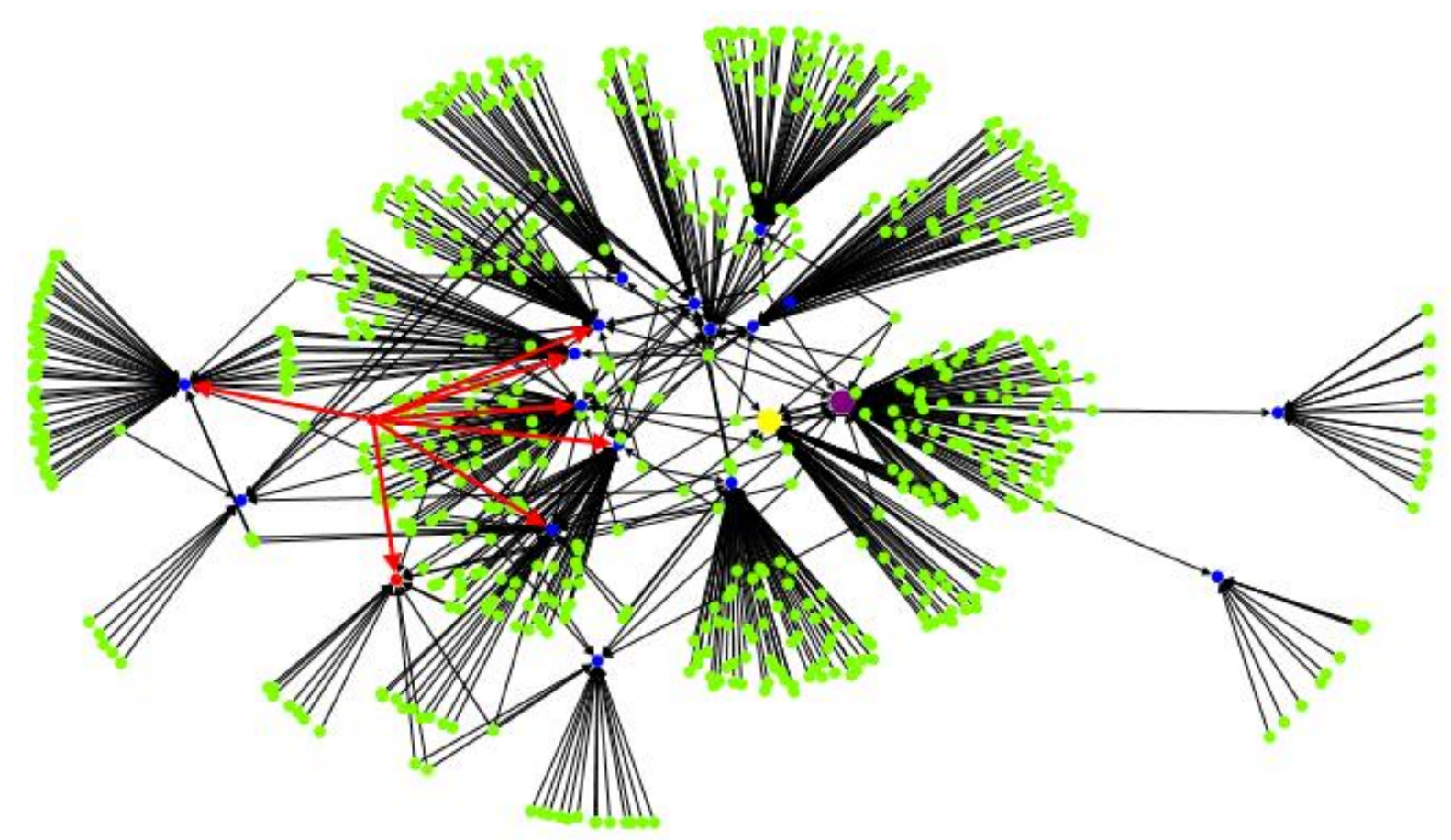

Figure 3: Diagram to show the relationship between contributor and projects. Each green dot represents a developer who contributes to the source code in one project and blue dot means each individual OSGeo project. Red arrows are a sample relationship that one developer contributes on seven different projects (see Table 2). Yellow and purple dots identify two projects, GeoTools and GeoServer. Because they have dependency relationships, we find many contributors boundary spans both projects. 


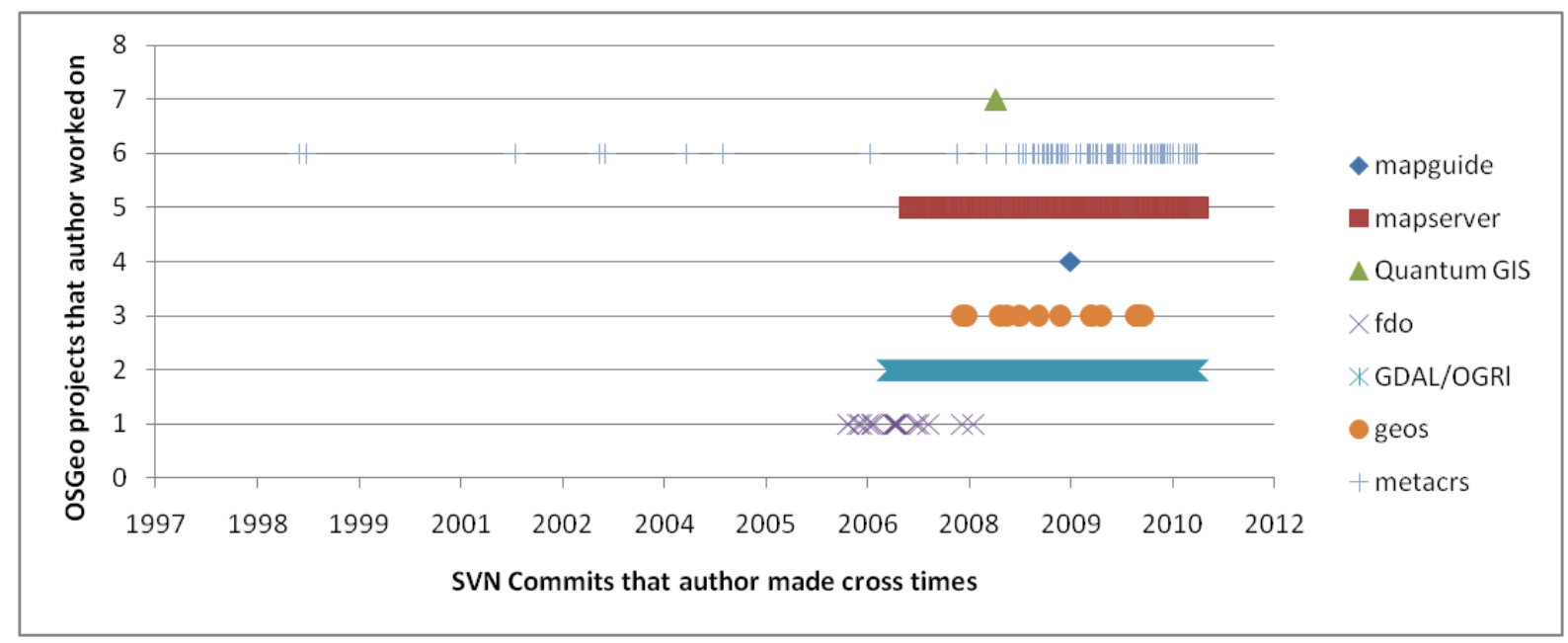

Figure 4: Boundary spanning diagram for a person who contributes on 7 OSGeo projects.

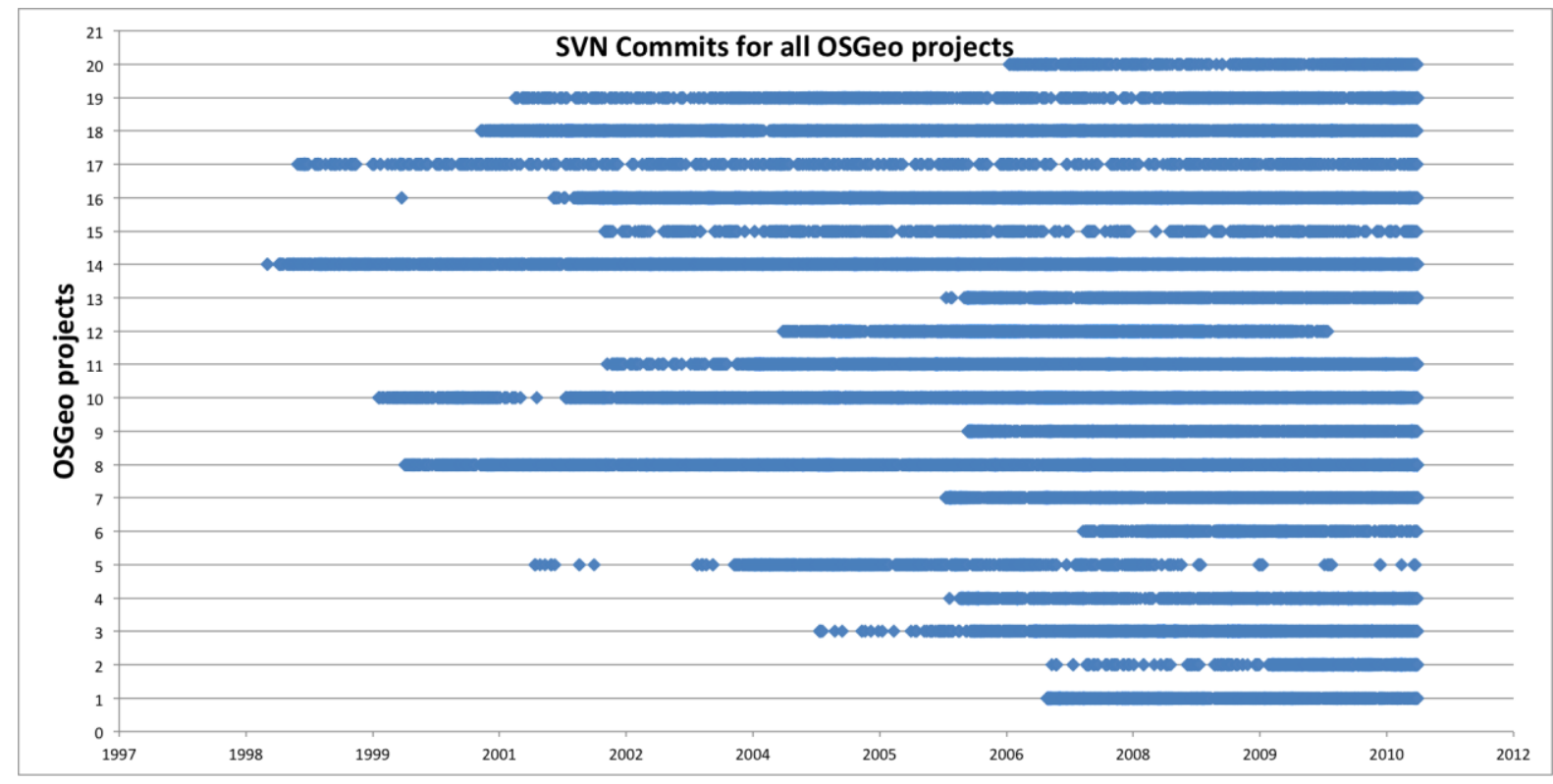

Figure 5: Diagram to show SVN Commits distribution for all studied mature OSGeo projects. 


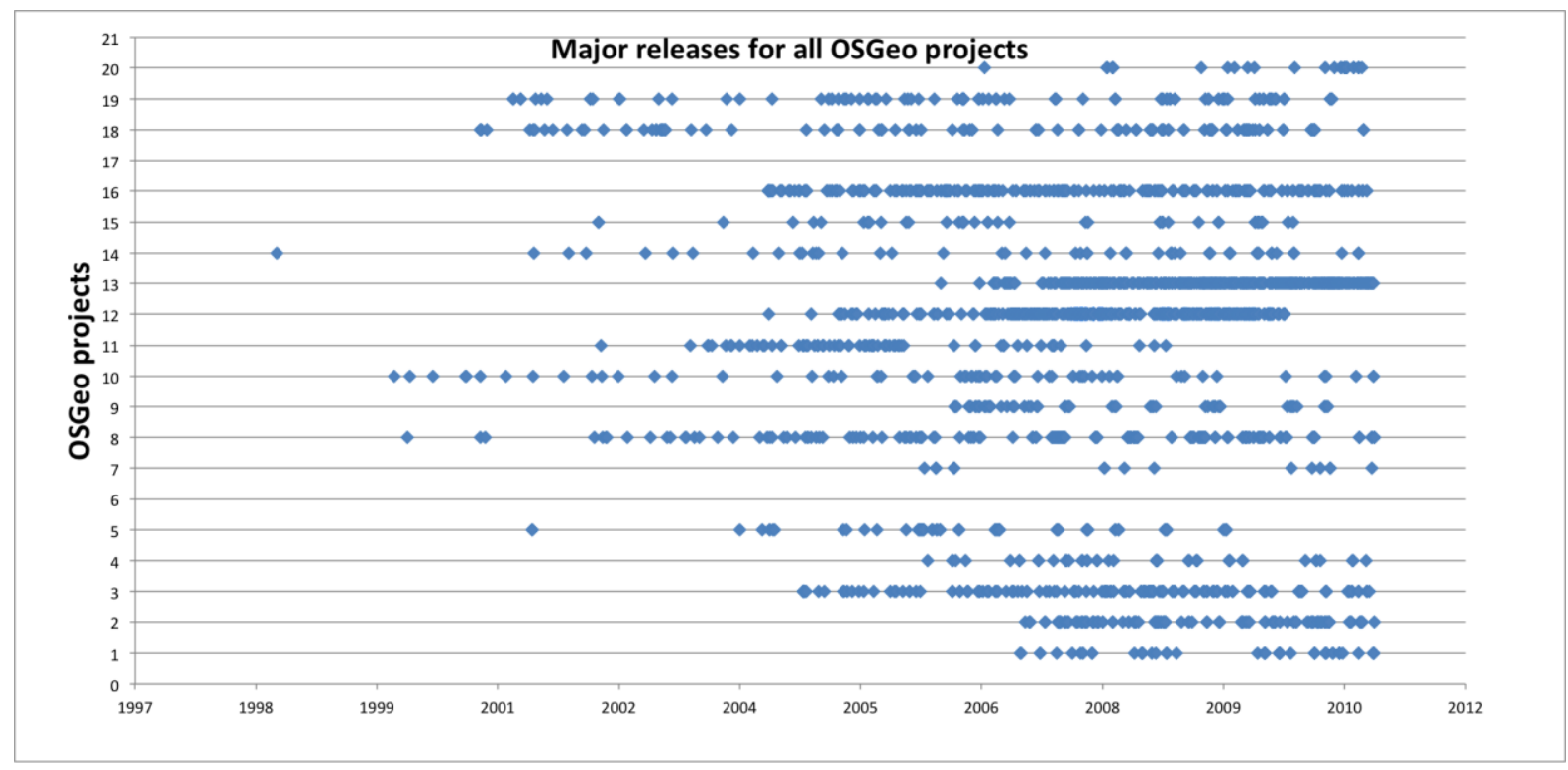

Figure 6: Distribution of releases of all OSGeo projects 
